\title{
Einführung in die Ergebnisdarstellung und -diskussion mit Überblick zum Wirkungsmodell zur Arbeitsgestaltung von Social Entrepreneuren
}

Im Rahmen der Datenauswertung wurde untersucht, welche Kategorien sich aus den Interviews herausarbeiten lassen, die zur Beantwortung der zentralen Forschungsfrage führen, und inwieweit diese Kategorien in einer Systematik dargestellt werden können. Prinzipiell ist denkbar, dass eine Systematik sowohl durch inhaltsbezogene Lagebeziehungen der Kategorien zueinander als auch durch deren Abgrenzung voneinander verdeutlicht werden kann (vgl. Kelle \& Kluge, 2010, S. 85 f., 91 ff.). Zur Beantwortung der zentralen Forschungsfrage konnten deutlich über 1.000 unterschiedliche Kodes nach der Reflexiven Grounded Theory herausgearbeitet und systematisch kategorisiert werden. Aus den dabei entwickelten Kategorien und der Art und Weise, wie die Kategorien zueinander in Verbindung stehen, setzt sich das in diesem Kapitel hergeleitete Wirkungsmodell zur Arbeitsgestaltung von Social Entrepreneuren (SE) zusammen, das alle Kategorien einbezieht und zu einem übergeordneten Ganzen vereint. Dieses Kapitel dient dazu, einen allgemeinen, einführenden Überblick zu dem Wirkungsmodell im Sinne einer Verständnisbasis zu schaffen.

Bei der vorliegenden Datenerhebung und -analyse erfüllten die in Kapitel 2 hergeleiteten Präkonzepte ihren Zweck, sich der Thematik annähern zu können. Allerdings erweist sich die dabei vorgenommene Differenzierung zwischen aktionsfeldbezogenen, kulturbezogenen, personenbezogenen und soziohierarchischen Besonderheiten nicht als sinnvolle Struktur zur Beantwortung der zentralen Forschungsfrage. Denn die in dieser Untersuchung herausgearbeiteten Dimensionen ergeben in ihrer Komplexität eine neue, zudem dynamische Gesamtstruktur, die geprägt ist von unterschiedlichen SE-spezifischen

Elektronisches Zusatzmaterial Die elektronische Version dieses Kapitels enthält Zusatzmaterial, das berechtigten Benutzern zur Verfügung steht https://doi.org/10.1007/978-3-658-35145-8_4. 
Belastungssituationen. Die differenzierte Auseinandersetzung mit diesen SEspezifischen Belastungssituationen stellt den inhaltlichen Kern bei der Darstellung und Diskussion der Untersuchungsergebnisse in Form des Wirkungsmodells zur Arbeitsgestaltung von SE dar.

Dabei ergeben sich zwei Besonderheiten für die Gesamtlogik zum Aufbau der Arbeit: Zum einen werden Diskussion und Ergebnisse miteinander verknüpft. Zum anderen erfolgt die Darstellung auf Basis des kodierten Datenmaterials in sechs Kapiteln (Kap. 5 bis Kap. 10). Bei dieser schrittweisen Darstellung mit Diskussion aller Wirkungsmodell-Einheiten wird das Verständnis zur Wirkungsweise von SE-spezifischen Belastungssituationen im Bedingungsgefüge systematisch hergeleitet und erläutert. Dieses Vorgehen wird dem hohen Komplexitätsgrad der Ergebnisse didaktisch bestmöglich gerecht.

Jedes dieser Kapitel widmet sich einer eigenständigen thematischen Auseinandersetzung und besitzt somit seinen eigenen Stellenwert im Gesamtgefüge der Merkmale und Bedingungen zur Arbeitsgestaltung von SE und zu deren Wirkung. Einerseits bildet damit jedes dieser Kapitel eine inhaltliche Einheit. Andererseits stehen Inhalte der einzelnen Kapitel zueinander in Verbindung. Diese Verbindungen können in der Übersicht als gemeinsame Gesamtstruktur abgebildet werden. Auf diese Weise entsteht in der Gesamtbetrachtung ein ganzheitliches Wirkungsmodell zur Arbeitsgestaltung von SE, dem sich alle im Rahmen der Datenerhebung herausgearbeiteten Kodes schlüssig zuordnen lassen.

Arbeitswissenschaftliche Theorien und Erkenntnisse werden zur Erklärung einzelner Untersuchungsergebnisse herangezogen. Wenngleich sich die Ergebnisse explizit auf Merkmale und Bedingungen zur Arbeitsgestaltung von SE in Deutschland konzentrieren, werden die gewonnenen Erkenntnisse zur Beantwortung der zentralen Forschungsfrage ebenfalls an internationalen Studien, die im Forschungskontext der Arbeitsgestaltung und der Wirkung von Arbeit von Social Entrepreneuren bestehen, gespiegelt und in der bestehenden Forschungslandschaft verortet. In Kapitel 2 dargestellte präkonzeptionelle Annahmen und Erkenntnisse werden hierbei berücksichtigt.

Bei mehreren Ergebnissen und Diskussionspunkten ist davon auszugehen, dass die aufgezeigten Phänomene zumindest teilweise in vergleichbarer Form auf KE zutreffen können, was i. d. R. in den Ausführungen thematisiert wird. Allerdings zeichnet sich dabei beispielsweise infolge der SE-spezifischen Infrastruktur eine eigene Dynamik innerhalb der Landschaft von Social Entrepreneurship Organisationen (SEO) ab und eigene Effekte können dabei zutagetreten, die dem übergeordneten Anspruchsdenken zum Abbau sozialer Benachteiligung widersprechen können. Aus diesem Grund erscheint es notwendig, die entsprechenden Ausführungen in der gewählten Genauigkeit zu diskutieren. 
Zur Illustration des Kategoriensystems werden Schilderungen der Interviewpartner zu generierten Kodes als Endnotenverweis eingearbeitet. Zwar hätten an vielen Stellen weitere Interviewzitate herangezogen werden können, so dass mit der exemplarischen Darstellung aus dem Datenmaterial kein Anspruch auf Vollständigkeit bestehen kann. Bei dem gegebenen umfangreichen Kode-Bestand und zum bestmöglichen Schutz der Interviewpartner wird sich auf Zitate konzentriert, die zur Verdeutlichung eines Ergebnisses und der Diskussion hinreichend passend erscheinen. Dennoch lassen sich im Rahmen der gegebenen inhaltlichen Überschneidungen, die das nochmalige Aufgreifen bereits thematisierter Ergebnisse erforderlich macht, weitere Kode-Beispiele einarbeiten. In der Gesamtbetrachtung werden alle Kodes eingearbeitet, die für das Beantworten der zentralen Forschungsfrage eine Relevanz besitzen.

Zunächst wird ein Überblick zum Aufbau dieses Wirkungsmodells gegeben, das ausschließlich aus den im Rahmen der Datenauswertung entwickelten Kodes hervorgegangen ist. Daraufhin erfolgen ein Kurzüberblick zu allen Kriterien und eine Verortung der Kriterien im Wirkungsmodell zur Arbeitsgestaltung von SE. Abbildung 4.1 stellt das Wirkungsmodell zur Arbeitsgestaltung grafisch dar.

\section{Struktureller Aufbau des Wirkungsmodells zu Arbeitsgestaltung von Social Entrepreneuren}

Sieben im Rahmen der Datenauswertung herausgearbeiteten Kategorien bilden Modellbausteine des Wirkungsmodells. Sie werden als Wirkungskriterien bezeichnet. Dabei handelt es sich um:

I. Wirkungskriterium I: SEO-Unterstützungsorganisationen (SEO-U)

II. Wirkungskriterium II: Politik/Gesetzgebung \& Reglementierungen

III. Wirkungskriterium III: Aufgabenbezogene Anforderungen/Besonderheiten

IV. Wirkungskriterium IV: Personenbezogene Merkmale von SE, Einstellungen \& Motive

V. Wirkungskriterium V: Arbeits- \& lernkulturelle Besonderheiten

VI. Wirkungskriterium VI: Beanspruchung bzw. Beanspruchungsgefüge

VII. Wirkungskriterium VII: Beanspruchungsfolgen

Das Wirkungskriterium VI „Beanspruchung bzw. Beanspruchungsgefüge“ bildet zugleich die Kernkategorie des Modells.

Die Wirkungskriterien I bis V verkörpern unterschiedliche Ist-Situationen für Belastungen, denen ein SE ausgesetzt sein kann. Jedes dieser Wirkungskriterien 


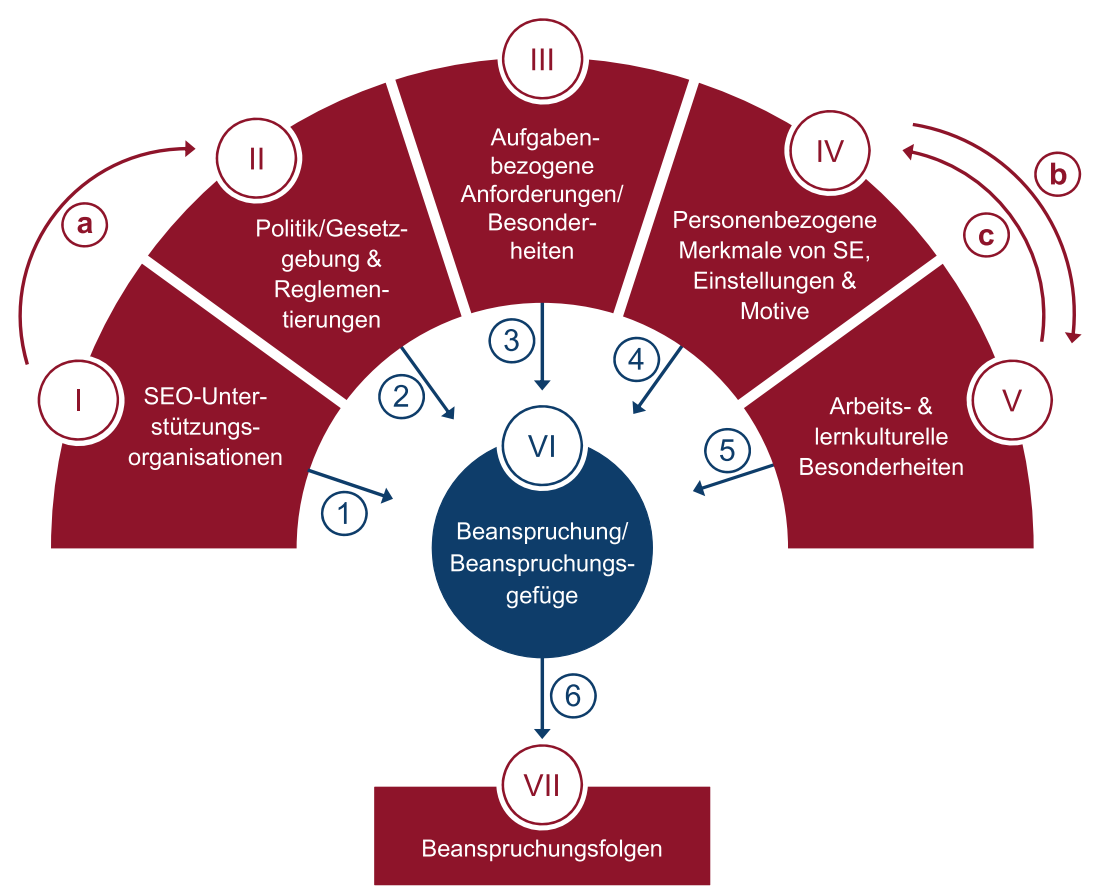

Abbildung 4.1 Wirkungsmodell zur Arbeitsgestaltung von Social Entrepreneuren. (eigene Darstellung)

spiegelt die gegebenen Infrastrukturen bzw. Merkmalsausprägungen wider. IstSituationen geben aber noch nicht zu erkennen, welches Belastungserleben und welche Beanspruchung aus den gegebenen Strukturen und Merkmalen hervorgehen. Demzufolge lässt sich die Reichweite der Bedeutung von Ist-Situationen nicht auf Basis der Wirkungskriterien erfassen, sondern nur prozessbasiert, also dynamisch.

Vergleichbar verhält es sich mit dem Wirkungskriterium VI: Denn aus dem gegebenen Beanspruchungsgefüge zeigen sich erst in der weiteren Entwicklung bestimmte Beanspruchungsfolgen.

Aus den Wirkungskriterien leiten sich insgesamt neun unterschiedliche Prozesse mit jeweils eigenen Wirkungsdynamiken ab. Sie lassen sich daher als Wirkungspfade charakterisieren.

Sechs der neun Wirkungspfade stehen im direkten Kontakt zur Kernkategorie: 
1. Wirkungspfad 1: Aktivitäten von SEO-Unterstützungsorganisationen (SEO-U) wirken als Belastungen beanspruchend und somit auf das Beanspruchungsgefüge von SE.

2. Wirkungspfad 2: Aktivitäten von Politikern und Behörden wirken als Belastungen beanspruchend und somit auf das Beanspruchungsgefüge von SE.

3. Wirkungspfad 3: Aufgabenbezogene Anforderungen wirken als Belastungen beanspruchend und somit auf das Beanspruchungsgefüge von SE.

4. Wirkungspfad 4: Personenbezogene Merkmale von SE, Einstellungen und Motive wirken als Belastungen beanspruchend und somit auf das Beanspruchungsgefüge von SE.

5. Wirkungspfad 5: Arbeits- und lernkulturelle Besonderheiten wirken als Belastungen beanspruchend und somit auf das Beanspruchungsgefüge von SE.

6. Wirkungspfad 6: Aus dem Beanspruchungsgefüge resultieren Beanspruchungsfolgen.

Darüber hinaus zeigen sich folgende drei Neben-Wirkungspfade, die keine direkte Verbindung zur Kernkategorie aufweisen, aber dennoch in SE-spezifischer Weise von Bedeutung sind:

7. Wirkungspfad a: Aktivitäten von SEO-Unterstützungsorganisationen wirken auf politische Rahmenbedingungen/die Gesetzgebung und Regelwerke. Da politische Rahmenbedingungen, die Gesetzgebung und Reglementierungen zum einen als Wirkungskriterium I und zum anderen im Wirkungspfad 2 auf das Beanspruchungsgefüge von SE wirken, ergibt sich eine separat zu benennende Einflussnahme, die zwar indirekt, jedoch in spezifischer Weise Auswirkungen auf das Beanspruchungsgefüge von SE haben kann.

8. Wirkungspfad b: Personenbezogene Merkmale von SE, Einstellungen und Motive wirken auf arbeits- und lernkulturelle Besonderheiten. Da arbeits- und lernkulturelle Besonderheiten zum einen als Wirkungskriterium $V$ und zum anderen im Wirkungspfad 5 auf das Beanspruchungsgefüge von SE wirken, ergibt sich eine separat zu benennende Dynamik, die zwar ebenfalls indirekt, jedoch in spezifischer Weise Auswirkungen auf das Beanspruchungsgefüge von SE haben kann.

9. Wirkungspfad c: Arbeits- und lernkulturelle Besonderheiten wirken auf personenbezogene Merkmale von SE, Einstellungen und Motive. Da personenbezogene Merkmale von SE, Einstellungen und Motive als Wirkungskriterium IV und im Wirkungspfad 4 auf das Beanspruchungsgefüge von SE wirken, ergibt sich abermals eine separat zu benennende Dynamik, die zwar ebenfalls indirekt, 
jedoch in spezifischer Weise Auswirkungen auf das Beanspruchungsgefüge von SE haben kann.

Es ist darauf hinzuweisen, dass keine genaue Trennschärfe zwischen den einzelnen Kategorien bestehen kann. Beispielsweise lassen sich Kodes zum Teil sowohl einem Wirkungskriterium als auch einem daraus hervorgehenden Wirkungspfad zuordnen. Hierbei wird eine Zuordnung der Zweckmäßigkeit halber und bei dem gegebenen Erkenntnisinteresse als kontingente Entscheidung getroffen; deren Darstellung lässt sich mit dem Bedeutungsgehalt der einzelnen Punkte nachvollziehen.

Die meisten Kategorien stehen untereinander in Verbindung und in Wechselwirkung zueinander. Dabei lassen sich allgemeingültige Merkmale der Arbeitsgestaltung wie z. B. der Arbeitsumgebung und der Arbeitsorganisation benennen (vgl. hierzu z. B. Schaper, 2019; Kaehler, 2020; Wieland, 2006). Über die beschriebenen neun Wirkungspfade hinaus lassen sich auf Basis des gewonnenen Datenmaterials keine Erkenntnisse ableiten, die im Besonderen auf SE zutreffen und die sich den benannten Wirkungspfaden nicht zuordnen lassen. Da sich die vorliegende Arbeit den Besonderheiten bei SE widmet, werden Verbindungen, denen das SE-Typische fehlt, nicht mit aufgenommen.

Trotz der damit gegebenen Einschränkungen wird die Zielsetzung, die Forschungsfrage unter Einbezug aller relevanten Kriterien im Gesamtverständnis zu beantworten, nicht beeinträchtigt.

Jedes Wirkungskriterium besteht wiederum aus mehreren Bestandteilen i. S. v. Teilkriterien. Jedes Teilkriterium setzt sich zusammen aus Kodes, die mit der Datenauswertung herausgearbeitet werden konnten. Sämtliche herausgearbeiteten Kodes lassen sich den Teilkriterien zuordnen.

Anhang $\mathrm{N}$ im elektronischen Zusatzmaterial zeigt als tabellarische Auflistung sämtliche Teilkriterien in Zuordnung zu ihren Wirkungskriterien.

In den folgenden Kapiteln 5 bis 10 werden der Reihe nach die sieben Wirkungskriterien mit ihren zugehörigen Wirkungspfaden dargestellt und diskutiert. Lediglich die Darstellung und Diskussion der Wirkungskriterien VI (Beanspruchung bzw. Beanspruchungsgefüge) und VII (Beanspruchungsfolgen) erfolgt gemeinsam in Kapitel 10. Dabei wird der Stellenwert von Belastungen, von Beanspruchung und Beanspruchungsfolgen dargestellt und diskutiert. 


\section{Literaturverzeichnis}

Kaehler, B. (2020). Komplementäre Führung. Ein praxiserprobtes Modell der Personalführung in Organisationen (3., vollständig überarbeitete Auflage). Wiesbaden: Springer Gabler.

Kelle, U. \& Kluge, S. (2010). Vom Einzelfall zum Typus. Fallvergleich und Fallkontrastierung in der qualitativen Sozialforschung (2., überarbeitete Auflage). Wiesbaden: VS Verlag für Sozialwissenschaften \& Springer Fachmedien.

Schaper, N. (2019). Arbeitsgestaltung in Produktion und Verwaltung. In: F. Nerdinger, G. Blickle und N. Schaper (Hrsg.), Arbeits- und Organisationspsychologie (4., vollständig überarbeitete Auflage). Berlin: Springer, S. 411-434.

Wieland, R. (Hrsg.) (2006). Themenschwerpunkt Betriebliches Gesundheitsmanagement. In: Wuppertaler Beiträge zur Arbeits- und Organisationspsychologie (1), Bergische Universität Wuppertal.

Open Access Dieses Kapitel wird unter der Creative Commons Namensnennung 4.0 International Lizenz (http://creativecommons.org/licenses/by/4.0/deed.de) veröffentlicht, welche die Nutzung, Vervielfältigung, Bearbeitung, Verbreitung und Wiedergabe in jeglichem Medium und Format erlaubt, sofern Sie den/die ursprünglichen Autor(en) und die Quelle ordnungsgemäß nennen, einen Link zur Creative Commons Lizenz beifügen und angeben, ob Änderungen vorgenommen wurden.

Die in diesem Kapitel enthaltenen Bilder und sonstiges Drittmaterial unterliegen ebenfalls der genannten Creative Commons Lizenz, sofern sich aus der Abbildungslegende nichts anderes ergibt. Sofern das betreffende Material nicht unter der genannten Creative Commons Lizenz steht und die betreffende Handlung nicht nach gesetzlichen Vorschriften erlaubt ist, ist für die oben aufgeführten Weiterverwendungen des Materials die Einwilligung des jeweiligen Rechteinhabers einzuholen. 